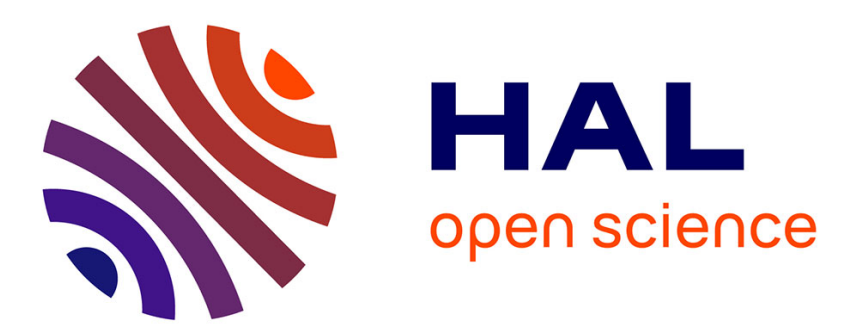

\title{
Etude de la dynamique en régimes quasiharmonique et anharmonique de la phase basse température de la triéthylènediamine par diffusion cohérente inélastique des neutrons
}

Alain Hédoux, J.L. Sauvajol, M. More

\section{To cite this version:}

Alain Hédoux, J.L. Sauvajol, M. More. Etude de la dynamique en régimes quasiharmonique et anharmonique de la phase basse température de la triéthylènediamine par diffusion cohérente inélastique des neutrons. Journal de Physique, 1988, 49 (2), pp.269-280. 10.1051/jphys:01988004902026900 . jpa-00210693

\section{HAL Id: jpa-00210693 https://hal.science/jpa-00210693}

Submitted on 1 Jan 1988

HAL is a multi-disciplinary open access archive for the deposit and dissemination of scientific research documents, whether they are published or not. The documents may come from teaching and research institutions in France or abroad, or from public or private research centers.
L'archive ouverte pluridisciplinaire HAL, est destinée au dépôt et à la diffusion de documents scientifiques de niveau recherche, publiés ou non, émanant des établissements d'enseignement et de recherche français ou étrangers, des laboratoires publics ou privés. 


\title{
Etude de la dynamique en régimes quasiharmonique et anharmonique de la phase basse température de la triéthylènediamine par diffusion cohérente inélastique des neutrons
}

\author{
A. Hedoux $\left({ }^{1}\right)$, J. L. Sauvajol $\left({ }^{2}\right)$ et M. More $\left({ }^{3, *}\right)$ \\ ( $\left.{ }^{1}\right)$ Laboratoire de Chimie Physique du Solide (UA 453), Ecole Centrale des Arts et Manufactures, \\ 92290 Châtenay-Malabry, France \\ ${ }^{2}$ ) G.D.P.C. (LA 233) Laboratoire d'Infrarouge, Université des Sciences et Techniques du Languedoc, place \\ E. Bataillon, 34060 Montpellier Cedex, France \\ $\left(^{3}\right)$ Laboratoire Léon Brillouin, CEN Saclay, 91191 Gif sur Yvette Cedex, France
}

(Reçu le 30 juin 1987, accepté le 19 octobre 1987)

\begin{abstract}
Résumé. - La triéthylènediamine $\mathrm{N}\left(\mathrm{CH}_{2} \mathrm{CH}_{2}\right)_{3} \mathrm{~N}$ cristallise jusqu'à $T_{\mathrm{c}}=351 \mathrm{~K}$ dans une phase solide ordonnée de symétrie hexagonale compacte. Les expériences de diffusion cohérente inélastique des neutrons réalisées dans la phase basse température s'inscrivent dans le cadre de l'étude du processus de la transition martensitique à $T_{\mathrm{c}}$ à partir de l'évolution avec la température des paramètres dynamiques. Ainsi les courbes de dispersion ont été déterminées à $190 \mathrm{~K}, 245 \mathrm{~K}$ et $295 \mathrm{~K}$. Les mesures effectuées à température ambiante ont permis d'ajuster les courbes de dispersion théoriques, calculées suivant un modèle quasi harmonique. Une étude de l'anharmonicité des modes de libration a été réalisée jusqu'à $T_{\mathrm{c}}$ en plusieurs points de la zone de Brillouin afin de décrire les mouvements susceptibles de piloter la transition h.c.p. $\rightarrow$ f.c.c. à $T_{c}$.

Abstract. - Triethylenediamine $\mathrm{N}\left(\mathrm{CH}_{2} \mathrm{CH}_{2}\right)_{3} \mathrm{~N}$ grows up to $T_{\mathrm{c}}$ in hexagonal compact ordered solid phase. Coherent inelastic neutron scattering in the low temperature phase has been used to understand the martensitic transition process at $T_{\mathrm{c}}$ from the evolution with temperature of the dynamical parameters. Thus, dispersion curves have been determined at $190 \mathrm{~K}, 245 \mathrm{~K}$ and $295 \mathrm{~K}$. Measurements at room temperature have allowed to fit dispersion curves with a quasi-harmonic model. Then anharmonicity of librational phonons has been studied up to $T_{\mathrm{c}}$ in several points of the Brillouin zone, in order to describe the motions which could possibly drive the h.c.p. $\rightarrow$ f.c.c. transition at $T_{\mathrm{c}}$.
\end{abstract}

\section{Introduction.}

Les transitions de phase ordre-désordre interviennent généralement entre une structure ordonnée de basse température de faible symétrie et une phase haute température à désordre orientationnel dont la structure moyenne est de haute symétrie. L'originalité des composés appartenant à la famille des bicyclo(222)-octanes $\left[\mathrm{X}\left(\mathrm{CH}_{2}-\mathrm{CH}_{2}\right)_{3} \mathrm{Y} ; \mathrm{X}, \mathrm{Y}=\mathrm{N}\right.$, $\mathrm{CH}]$ est de posséder une phase basse température de haute symétrie (hexagonale), la structure moyenne de la phase désordonnée étant cubique à faces centrées. De plus, compte tenu de la symétrie d'ordre trois de la molécule et de la symétrie

(*) Adresse actuelle: Laboratoire de Dynamique des Cristaux Moléculaires (UA 801), Université des Sciences et Techniques de Lille, 59655 Villeneuve d'Ascq Cedex, France. hexagonale du réseau basse température, l'activation d'un désordre uniaxial moléculaire à l'approche de la transition martensitique h.c.p. $\rightarrow$ f.c.c. à $T_{c}$ est attendue.

$\mathrm{La}$ triéthylènediamine $\left[\mathrm{N}\left(\mathrm{CH}_{2} \mathrm{CH}_{2}\right)_{3} \mathrm{~N}\right.$ (Fig. 1)

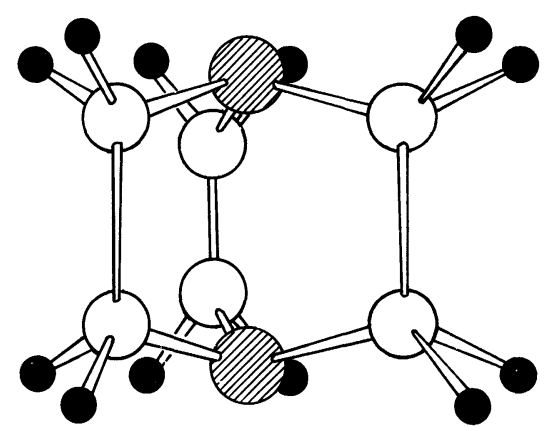

Fig. 1. - Squelette de la molécule de TEDA. [Skeleton of the TEDA molecule.] 
dénommée par la suite TEDA] présente l'intérêt supplémentaire par rapport aux composés de la même famille (bicyclo(222)-octane, quinuclidine), de cristalliser à la température ambiante dans sa phase ordonnée [groupe d'espace $\mathrm{P}_{3} / m, Z=2$ ] (Fig. 2). Ainsi l'étude de la transition de phase du $1^{\text {cr }}$ ordre (à $\left.T_{\mathrm{c}}=351 \mathrm{~K}\right)$ à partir de l'évolution avec la température des paramètres statiques et dynamiques de la phase ordonnée est alors possible.
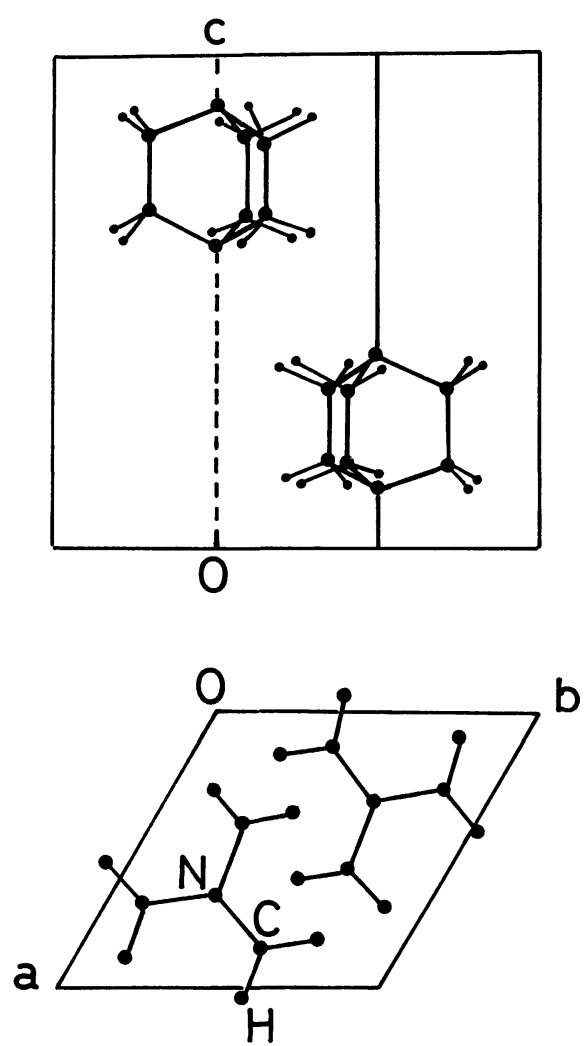

Fig. 2. - Représentation de la maille élémentaire de TEDA dans les plans $(\mathbf{b}, \mathbf{c})$ et $(\mathbf{a}, \mathbf{b})$.

[Representation of the TEDA unit cell in the planes $(\mathbf{b}, \mathbf{c})$ and $(\mathbf{a}, \mathbf{b})$.]

Les premiers renseignements obtenus dans le cadre de cette étude proviennent de données de résonance magnétique nucléaire [1], de diffraction des rayons $\mathrm{X}[2]$ et de diffusion Raman de la lumière $[3,4]$. Les mesures de résonance magnétique nucléaire s'interprètent en tenant compte d'une réorientation moléculaire autour de l'axe d'ordre 3 $\left(\mathrm{C}_{3}\right)$ de la molécule entre positions indiscernables (angles de saut de $120^{\circ}$ ). Ce processus est dominant au-dessus de $190 \mathrm{~K}$ et jusqu'à $T_{\mathrm{c}}$ où la réorientation générale apparaît [1]. D'autre part les données de diffraction $\mathrm{X}$ à différentes températures mettent en évidence une forte amplitude de la libration autour de $\mathrm{C}_{3}$ à haute température. L'amplitude de cette libration croît avec la température plus fortement que celle des librations perpendiculaires à $\mathrm{C}_{3}$ [2].
Enfin les mesures de diffusion Raman de la lumière montrent à la fois la forte anharmonicité des modes de libration et la contribution du désordre uniaxial dans l'évolution avec la température des largeurs des modes correspondants $\left(\mathrm{A}_{\mathrm{g}}, \mathrm{E}_{1 \mathrm{~g}}\right)$ [4]. L'ensemble de ces résultats s'explique d'une manière cohérente en supposant que la réorientation générale de la molécule à $T_{\mathrm{c}}$ est précédée par une réorientation moléculaire uniaxiale entre positions indiscernables qui pourrait se produire via une orientation métastable à $60^{\circ}$ des positions d'équilibre. Cette description est en accord avec les calculs de potentiel d'interaction entre molécules dans la phase II de TEDA effectués par Reynolds [5].

Le but de cet article est d'affiner la description de la dynamique des mouvements moléculaires dans la phase II de TEDA à partir des données de diffusion cohérente des neutrons. Dans un premier temps nous comparerons les courbes de dispersion des phonons et librons (à $T=295 \mathrm{~K}$ ) à celles calculées à partir d'un modèle dynamique quasi harmonique. Dans un deuxième temps nous étudierons les variations des courbes de dispersion en fonction de la température et en particulier les évolutions des fréquences $\omega_{i}(\mathbf{q})$ et des largeurs $\Gamma_{i}(\mathbf{q})$ des modes de libration afin de séparer la part due à l'anharmonicité de celle due à une activation éventuelle d'un désordre uniaxial.

\section{Conditions expérimentales.}

2.1 LE CRISTAL DE N $\left(\mathrm{CD}_{2} \mathrm{CD}_{2}\right)_{3} \mathrm{~N}$. - La triéthylènediamine deutériée est obtenue à partir de la triéthylènediamine hydrogénée (produit Merck) par une méthode d'échange sous pression et à haute température $\left(t=130^{\circ} \mathrm{C}\right)$ dans un autoclave. TEDA étant un composé fortement volatil et hygroscopique le cristal doit être fabriqué et conservé dans un conteneur fermé. L'échantillon que nous avons utilisé a été obtenu dans une sphère de quartz (diamètre $10 \mathrm{~mm}$ ) par une méthode de transport en phase vapeur à partir d'un produit deutérié à $98 \%$.

Lors de l'expérience de diffusion neutronique la température de l'échantillon doit pouvoir prendre des valeurs comprises entre 200 et $350 \mathrm{~K}$. Des expériences préliminaires ont montré que la pression exercée sur les parois du conteneur pour des températures entre 330 et $340 \mathrm{~K}$ était susceptible de briser l'ampoule de quartz et à partir de là déstabiliser le cristal dans son enceinte. Nous avons donc été amenés à doubler l'enceinte de quartz par une enceinte d'aluminium étanche dans laquelle le tube de quartz est ouvert. Ce dispositif a permis d'effectuer toutes les mesures jusqu'à la température de transition sans ennuis.

2.2 Collection et TRAitement DES DONNÉES. $\mathrm{Au}$ cours des expériences nous avons été confrontés à deux problèmes spécifiques : 
a) la forte anharmonicité de certains modes de libration autour de l'axe $\mathrm{C}_{3}$ conduit à des groupes de phonons très larges ;

b) certains modes optiques de translation ou (et) de libration ont des fréquences voisines et apparaissent groupés dans la zone de Brillouin ce qui peut donner lieu à des recouvrements notables.

L'effet conjugué de ces deux phénomènes impose si l'on souhaite une précision suffisante dans la détermination des fréquences et des largeurs des modes, d'une part de choisir une résolution instrumentale adaptée et d'autre part de traiter numériquement les spectres obtenus pour tenir compte à la fois de la résolution instrumentale nécessairement limitée et des recouvrements de modes possibles.

L'expérience a été réalisée au réacteur ORPHÉE du Laboratoire Léon Brillouin (CEN Saclay) sur les spectromètres à 3 axes 4F2, 4F1 installés sur un faisceau de neutrons froids. Pour la plupart des scans, nous avons utilisé des neutrons de longueur d'onde $3,49 \AA\left(k_{i}=1,8 \AA^{-1}\right)$ avec des collimations horizontales effectives de $60^{\prime} / 40^{\prime} / 20^{\prime} / 20^{\prime}$ et des collimations verticales naturelles estimées à : $1^{\circ}, 1,5^{\circ}, 2^{\circ}$, $4^{\circ}$. Monochromateurs (double) et analyseur sont en graphite pyrolitique (réflexion 002) courbés verticalement pour un des monochromateurs et plats pour le $2^{\mathrm{c}}$ monochromateur et l'analyseur. La diffusion inélastique a été mesurée autour du centre de zone $(3 ; 0 ;-3)$, au bord de zone $(0 ; 0 ;-4,5)$ et en quelques points intérieurs à la zone comme $(3 ; 0$; $-3,2),(1 ; 0 ;-4,2) \ldots$ Ces valeurs de $\mathbf{Q}$ correspondent à des facteurs de forme favorables pour les phonons qui nous intéressent. La température a été variée de 190 à $350 \mathrm{~K}$. Les variations des paramètres de la maille avec la température sont représentées sur la figure 3 .

Les conditions de mesure ainsi définies (longueur d'onde et collimation) sont un optimum permettant d'atteindre les zones de Brillouin déjà citées et d'obtenir des phonons d'intensité raisonnable. Cependant la résolution (\# $0,08 \mathrm{Thz}$ FWHM pour un mode non dispersif) est insuffisante pour la plupart des cas où un ou deux modes (ou plus) sont actifs simultanément dans une bande d'énergie de l'ordre de $1 \mathrm{Thz}$. Il est alors nécessaire de faire appel à des programmes numériques de simulation

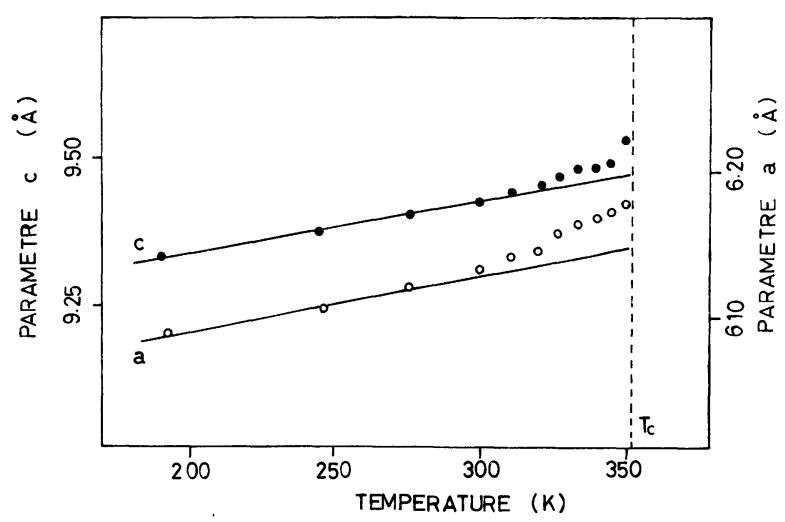

Fig. 3. - Variations avec la température des paramètres de la maille.

[Lattice parameters versus temperature.]

(RESO) ou d'affinement (FIT), utilisables «en ligne » depuis l'expérience. La plupart des modes ont été ajustés à partir de la fonction de diffusion de l'oscillateur harmonique amorti :

$$
F_{Q}(\omega) \simeq \frac{1}{\pi} \frac{\Gamma \cdot \omega_{Q}^{2}}{\left(\omega^{2}-\omega_{Q}^{2}\right)^{2}+\omega^{2} \cdot \Gamma^{2}}
$$

où $\omega_{Q}$ est la fréquence caractéristique du mode mesuré au vecteur de diffusion $Q$ et $\Gamma$ son amortissement. Cette fonction de diffusion est convoluée avec la résolution instrumentale de l'appareil suivant un schéma analogue à celui utilisé dans la référence [6].

Le choix du plan de diffusion $\left(\mathbf{a}^{*}, \mathbf{c}^{*}\right)$ a permis de mesurer la quasi-totalité des courbes de dispersion pour la direction $\mathbf{c}^{*}$ du réseau réciproque et les branches acoustiques pour la direction $\mathbf{a}^{*}$ du réseau réciproque et cela à trois températures : 190,245 , $290 \mathrm{~K}$. La figure 4 montre l'ensemble des courbes pour $T=295 \mathrm{~K}$. Les symboles représentés correspondent aux vecteurs de diffusion et aux symétries indiquées dans le tableau I. L'indexation des symétries a été effectuée en accord avec la définition des vecteurs $\mathbf{q}$ donnée figure 5 . Le diagramme de corrélation des symétries activées suivant la direction $\mathbf{c}^{*}$ est donnée dans le tableau II.

L'évolution avec la température des fréquences et des largeurs de plusieurs modes optiques (phonons et librons) en différents vecteurs $\mathbf{q}$ de la direction $\mathbf{c}^{*}$

Tableau I. - Polarisations et symétries des phonons dans la direction $\mathbf{c}^{*}$.

[Phonon polarizations and symmetries along $\mathbf{c}^{*}$.]

\begin{tabular}{|c|c|c|c|}
\hline Symbole & Zone & Symétries activées & Polarisation \\
\hline $\mathbf{\square}$ & $\left(\begin{array}{lll}0 & 0 & 4\end{array}\right)$ & $\Omega_{1}^{(1)}, \Delta_{1}^{(1,2)}$ & $\mathbf{q} / / \mathbf{Q}$, longitudinale \\
$\bullet$ & $\left(\begin{array}{lll}1 & 0 & 4\end{array}\right)$ & $\mathrm{E}_{2 u}, \Omega_{2}^{(2)}, \Omega_{3}^{(1,2)}, \Omega_{4}^{(1,2)}, \Delta_{4}^{(1,2)}$ & $\mathbf{q} / / \mathbf{Q}$, longitudinale \\
$*$ & $(4,0,0)$ & $\mathrm{E}_{2 u}, \mathrm{E}_{2 \mathrm{~g}}, \mathrm{~B}_{\mathrm{u}}, \mathrm{A}_{\mathrm{g}}, \mathrm{E}_{1 \mathrm{~g}}$ & $\mathbf{q} \perp \mathbf{Q}$, transverse \\
$\mathbf{\Delta}$ & $(3,0,3)$ & $\mathrm{E}_{2 \mathrm{u}}, \mathrm{E}_{2 \mathrm{~g}}, \mathrm{Bu}, \Omega_{2}^{(1)}, \Omega_{4}^{(1,2)}$ & $\mathbf{q} \perp \mathbf{Q}$, transverse \\
\hline
\end{tabular}




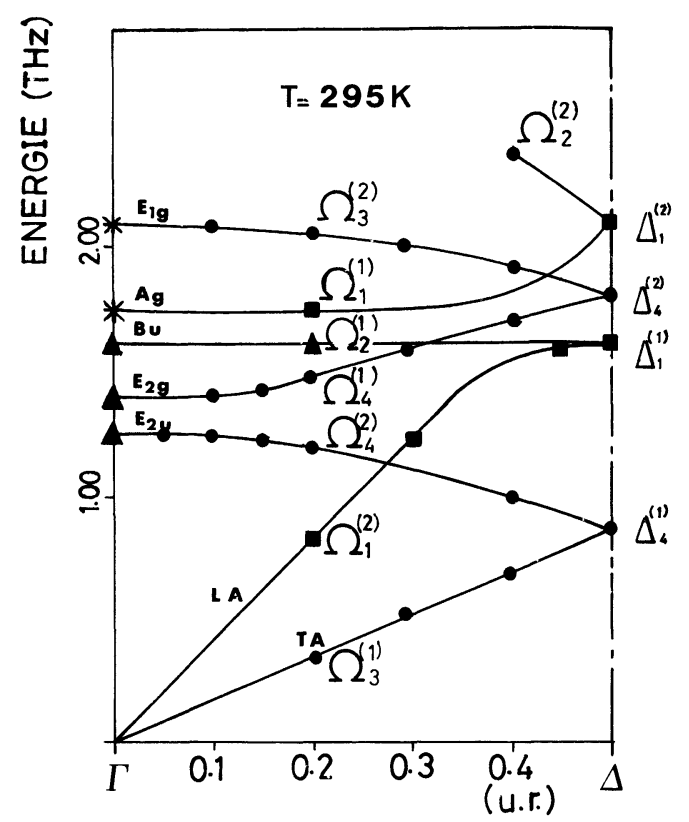

Fig. 4. - Courbes de dispersion expérimentale suivant la direction $\mathrm{c}^{*}$ à $T=295 \mathrm{~K}$.

[Experimental dispersion curves along $\mathbf{c}^{*}$ direction at 295 K.]

Tableau II. - Diagramme de corrélation des symétries suivant la direction $\mathrm{c}^{*}$.

[Correlation diagram along $\mathbf{c}^{*}$.]

$$
\begin{aligned}
& \operatorname{Ag}(1)-\Omega_{1}^{(1)}(1) \\
& \mathrm{Bu}(1)-\Omega_{2}^{(1)}(1)>\Delta_{1}^{(1)}(2) \\
& \begin{array}{l}
\mathrm{Au}(1)-\Omega_{1}^{(2)}(1) \succ \Delta_{1}^{(2)}(2) \\
\mathrm{Bg}(1)-\Omega_{2}^{(2)}(1) \succ \Delta^{(2)}
\end{array} \\
& \begin{array}{l}
\mathrm{E}_{2 \mathrm{~g}}(2)-\Omega_{4}^{(2)}(2) \\
\mathrm{E}_{1 \mathrm{u}}(2)-\Omega_{3}^{(1)}(2)
\end{array} \Delta_{2}^{(1)}(2), \Delta_{3}^{(1)}(2)=\Delta_{4}^{(1)}(4) \\
& \begin{array}{l}
\mathrm{E}_{1 \mathrm{~g}}(2)-\Omega_{3}^{(2)}(2) \\
\mathrm{E}_{2 \mathrm{u}}(2)-\Omega_{4}^{(1)}(2)
\end{array} \Delta_{2}^{(2)}(2), \Delta_{3}^{(2)}(2)=\Delta_{4}^{(2)}(4)
\end{aligned}
$$

$\Gamma=(0,0,0) \Omega=(0,0,2 \pi \eta / c) \Delta=(0,0, \pi / c)$

$\Delta_{2}$ et $\Delta_{3}$ sont complexes conjuguées et constituent les matrices de base d'une représentation $\Delta_{4}$ de degré 4 .

Les représentations irréductibles sont notées sous la forme :

$\Gamma_{j}^{(i)}(n)$ où $\Gamma_{j}$ est le nom de la représentation $n$ le degré de dégénérescence de la représentation d'indice $i$.

de la zone de Brillouin a été déterminée et cela jusqu'à la température de transition $T_{\mathrm{c}}$. Ces résultats seront présentés ultérieurement. Nous allons maintenant nous intéresser à la description des courbes de dispersion par un modèle approprié.

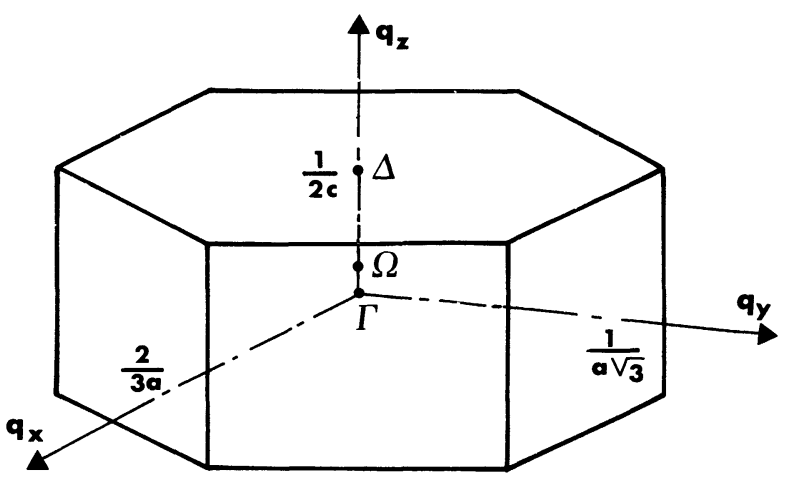

Fig. 5. - Définition des points étudiés suivant la direction

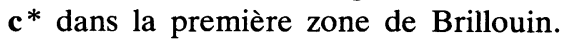

[Definition of studied points along $\mathbf{c}^{*}$ direction in the first Brillouin zone.]

\section{Calcul des courbes de dispersion à l'aide d'un modèle harmonique.}

Dans ce paragraphe nous indiquons la manière avec laquelle nous avons procédé en vue d'obtenir un ajustement des courbes de dispersion expérimentales par un modèle dynamique harmonique. La comparaison entre les courbes expérimentales et calculées sera faite à $T=295 \mathrm{~K}$. En effet c'est à cette température que nous possédons d'une part le plus de renseignements sur les coordonnées atomiques dans la maille et d'autre part le jeu de constantes d'interaction intermoléculaire rapportées dans la littérature a été déterminé à la température ambiante [7].

Dans l'hypothèse de molécules rigides acceptable dans le cas de TEDA dans la mesure où par exemple la spectrométrie Raman indique qu'aucun mode interne n'a de fréquence inférieure à $300 \mathrm{~cm}^{-1}$ chaque molécule possède 6 degrés de liberté : 3 relatifs à la translation d'ensemble de la molécule et 3 relatifs à la libration d'ensemble de la molécule, soit 12 degrés de liberté pour décrire les mouvements des deux molécules contenues dans la maille de TEDA. La théorie de la dynamique d'un cristal moléculaire a été complètement décrite par Venkataraman et Sahni [8]. Nous nous bornerons seulement à décrire les différentes étapes du calcul en explicitant certains termes dans le cas de TEDA. Dans ce formalisme, on définit une matrice dynamique, ici de dimension $12 * 12$, par l'expression :

$$
\begin{aligned}
B_{\alpha \beta}^{i i^{\prime}}\left(\mathbf{q}, \kappa \kappa^{\prime}\right)=\sum_{l^{\prime}} \phi_{\alpha \beta}^{i i^{\prime}}\left(0 \kappa, l^{\prime} \kappa^{\prime}\right) \times \\
\\
\times \exp \left\{i \mathbf{q}\left[\mathbf{X}\left(l^{\prime}\right)-\mathbf{X}(0)\right]\right\}
\end{aligned}
$$$$
\text { où : }
$$

- $\alpha, \beta$ sont les coordonnées cartésiennes

- $i, i^{\prime}$ réfèrent à des mouvements de translation $i\left(i^{\prime}\right)=t$ ou de libration $i\left(i^{\prime}\right)=r$

- $l, k$ indice une molécule $k$ de la maille $l$

- q est le vecteur d'onde

$-\phi_{\alpha \beta}^{i i^{\prime}}\left(\begin{array}{ll}0 & l^{\prime} \\ k & k^{\prime}\end{array}\right)$ sont des coefficients de couplage 
inter-moléculaire. Leurs symétries sont déduites de relations d'invariance sous l'effet des différentes opérations de symétrie du cristal ainsi que de la définition propre de ces coefficients.

Compte tenu de la symétrie de la phase basse température de TEDA et en se limitant aux interactions entre molécules premières et deuxièmes voisines, on peut déduire l'ensemble des constantes de force. Ces différents tenseurs peuvent être calculés numériquement en utilisant un potentiel d'interaction intermoléculaire $V\left(l k, l^{\prime} k^{\prime}\right)$. Les coefficients de couplage sont calculés à partir des dérivées premières et secondes de $V\left(l k, l^{\prime}, k^{\prime}\right)$ par rapport à $R^{2}\left(l k, l^{\prime} k^{\prime}\right)$, carré de la distance intermoléculaire. Nous avons choisi pour $V\left(l k, l^{\prime} k^{\prime}\right)$ un potentiel de type Buckingham qui décrit l'interaction entre deux molécules à partir des interactions entre atomes constituant les molécules :

$$
V\left(l k, l^{\prime} k^{\prime}\right)=\sum_{\substack{i j \\ i \in l k \\ j \in l^{\prime} k^{\prime}}}\left[-A / R_{i j}^{6}+B \exp \left(-C R_{i j}\right)\right]
$$

$A_{i j}, B_{i j}$ et $C_{i j}$ sont des paramètres se référant à un type d'interaction donné.

A partir de la symétrie des coefficients de couplage on peut dériver pour une direction particulière de $\mathbf{q}$, ici la direction $\mathrm{c}^{*}$, la forme symétrisée de la matrice dynamique.

La résolution de l'équation du mouvement,

$$
\omega^{2}(\mathbf{q}) m U(\mathbf{q})=B(\mathbf{q}) U(\mathbf{q})
$$

permet de connaître l'ensemble des 12 fréquences $\omega_{j}(q)$ associées aux modes de vibration de vecteurs propres $U_{j}(\mathbf{q})(j=1,12)$. $U(\mathbf{q})$ est la matrice de ces vecteurs propres et $m$ la matrice des «masses» définie par :

$$
\begin{aligned}
& m_{\alpha \beta}^{t t}\left(\kappa \kappa^{\prime}\right)=m_{\kappa} \delta_{\alpha \beta} \delta_{\kappa \kappa^{\prime}} \\
& m_{\alpha \beta}^{r r}\left(\kappa \kappa^{\prime}\right)=I_{\alpha \beta}(\kappa) \delta_{\kappa \kappa^{\prime}} \\
& m_{\alpha \beta}^{r t}\left(\kappa \kappa^{\prime}\right)=m_{\alpha \beta}^{t r}\left(\kappa \kappa^{\prime}\right)=0
\end{aligned}
$$

où $m_{k}$ est la masse et $I_{\alpha \beta}(k)$ le tenseur d'inertie de la molécule $k$.

L'ensemble des valeurs $\omega_{j}(\mathbf{q})$ calculées pour différentes valeurs de $q$ permet le tracé des courbes de dispersion (Fig. 6). La comparaison avec les courbes expérimentales (Fig. 4) montre que l'allure générale de ces courbes est décrite par le modèle, en particulier les valeurs des vitesses acoustiques mesurées et calculées sont en bon accord. Toutefois des écarts sensibles sont observés pour les modes optiques. Pour le centre de zone ce désaccord est visualisé dans le tableau III. Nous rappelons que ces résultats ont été obtenus sans aucun ajustement, en outre on peut penser à la vue de l'ensemble des résultats que le caractère anharmonique des modes optiques qui n'est pas pris en compte dans ce calcul est une

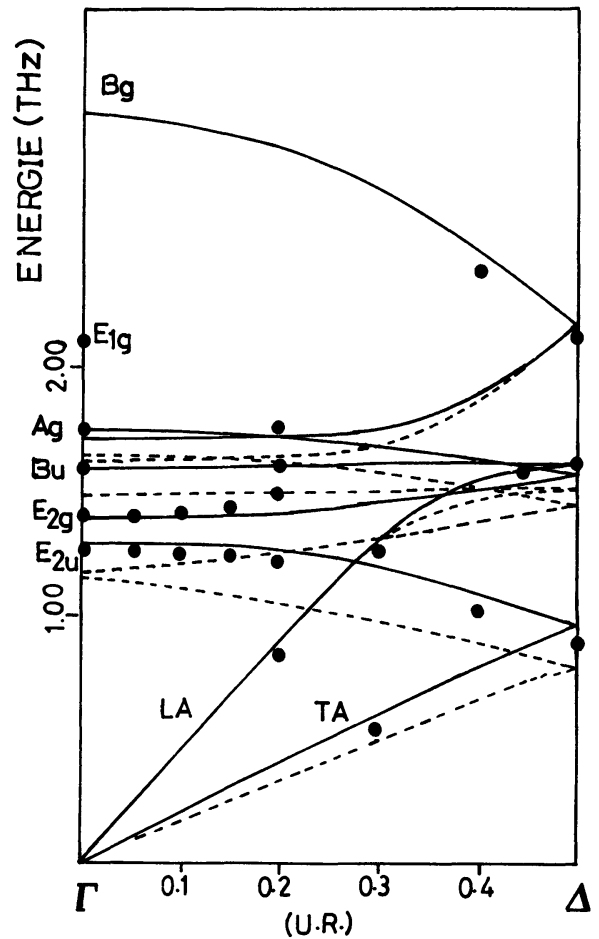

Fig. 6. - Courbes de dispersion calculées à température ambiante suivant la direction $\mathrm{c}^{*}$. a) Sans ajustement (trait discontinu). b) Après ajustement (trait plein). Les données expérimentales sont représentées par les points.

[Calculated dispersion curves (at room temperature) along c*. a) Without fit (dotted line). b) After a fit (full line). Full circles represent experimental data.]

Tableau III. - Comparaison entre les fréquences calculées (avec et sans ajustement) et expérimentales des modes optiques.

[Comparison between calculated (with and without a fit) and experimental frequencies of optical modes.]

\begin{tabular}{|l|c|c|c|}
\hline & Expérience & $\begin{array}{c}\text { Calcul } \\
\text { sans } \\
\text { ajustement }\end{array}$ & $\begin{array}{c}\text { Calcul } \\
\text { avec } \\
\text { ajustement }\end{array}$ \\
\hline $\mathrm{E}_{1 \mathrm{~g}}$ & $2,10 \mathrm{Thz}$ & $1,54 \mathrm{Thz}$ & $1,76 \mathrm{Thz}$ \\
$\mathrm{A}_{\mathrm{g}}$ & $1,76 \mathrm{Thz}$ & $1,62 \mathrm{Thz}$ & $1,72 \mathrm{Thz}$ \\
$\mathrm{B}_{\mathrm{u}}$ & $1,60 \mathrm{Thz}$ & $1,48 \mathrm{Thz}$ & $1,60 \mathrm{Thz}$ \\
$\mathrm{E}_{2 \mathrm{~g}}$ & $1,40 \mathrm{Thz}$ & $1,16 \mathrm{Thz}$ & $1,40 \mathrm{Thz}$ \\
$\mathrm{E}_{2 \mathrm{u}}$ & $1,26 \mathrm{Thz}$ & $1,14 \mathrm{Thz}$ & $1,30 \mathrm{Thz}$ \\
\hline
\end{tabular}

contribution qui ne peut être négligée même à $295 \mathrm{~K}$ (voir le paragraphe suivant).

Un ajustement des constantes de force $\phi$ a été effectué dans un deuxième temps afin d'obtenir un meilleur accord. En effet au centre et en bord de zone, la diagonalisation de la matrice dynamique $B(q)$ fait apparaître des relations simples entre certaines fréquences optiques et des termes des 
tenseurs qui peuvent être ainsi évalués directement à partir des fréquences expérimentales. Les nouvelles courbes de dispersion sont représentées figure 6. On note à l'évidence un meilleur accord, en particulier le phénomène «d'anti-crossing » des branches $E_{2 g}$ et $\mathrm{E}_{2 \mathrm{u}}$ près du centre de zone est clairement restitué. Cet accord apparaît dans la comparaison entre fréquences expérimentales et calculées des modes en centre de zone (Tab. III). On notera cependant que la branche optique $E_{1 \mathrm{~g}}$ en centre de zone ne peut être ajustée par cette méthode (pour cette branche aucune relation ne permet de déduire d'une manière univoque les différents termes du tenseur $\phi$ qui entrent dans l'expression analytique de $\omega_{\mathrm{E}_{1 \mathrm{~g}}}(\mathbf{q})$ ).

\section{Variation des courbes de dispersion avec la tempé-} rature.

4.1 COMPORTEMENT DES VITESSES ACOUSTIQUES. Quelques groupes de phonons acoustiques pour $T=295 \mathrm{~K}$ sont présentés figure 7. Les variations avec la température des vitesses acoustiques ont été tracées sur un intervalle allant de 190 à $310 \mathrm{~K}$ (Fig. 8a et 8b). Les variations linéaires observées

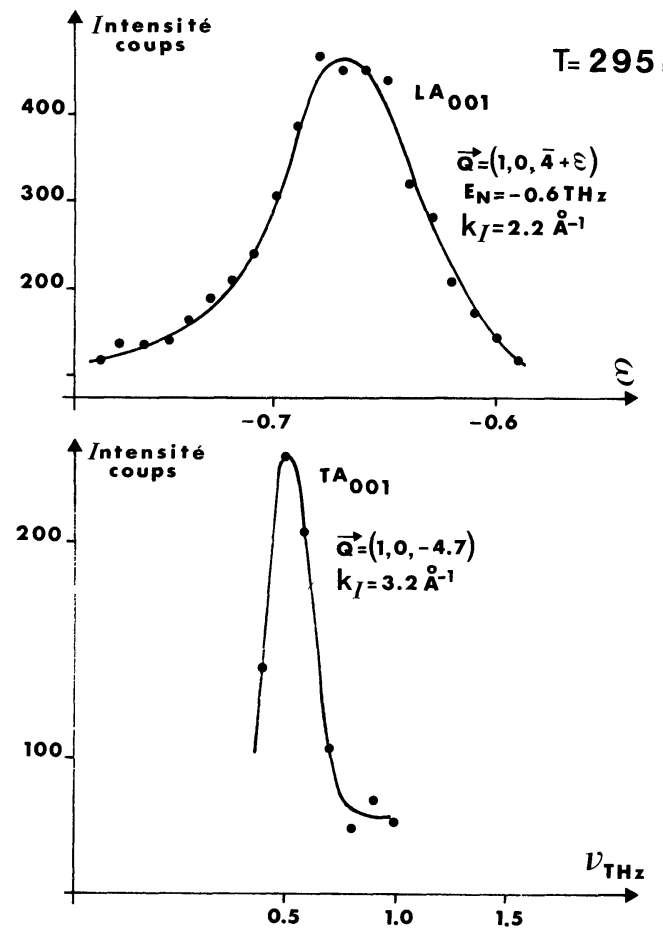

Fig. 7. - Exemples de phonons acoustiques à $295 \mathrm{~K}$.
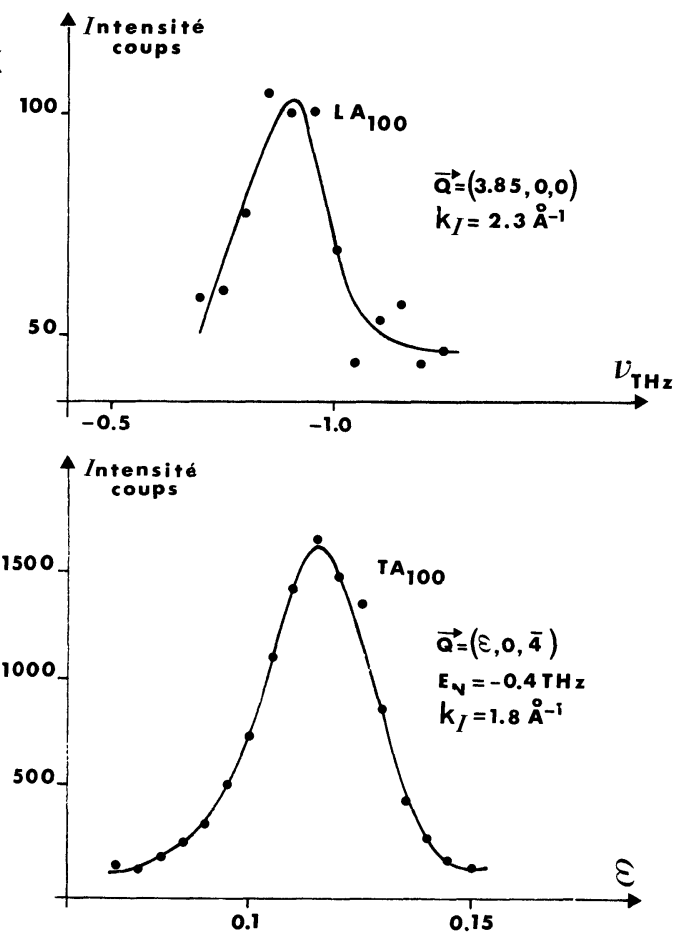

[Examples of acoustic phonons at $295 \mathrm{~K}$.]
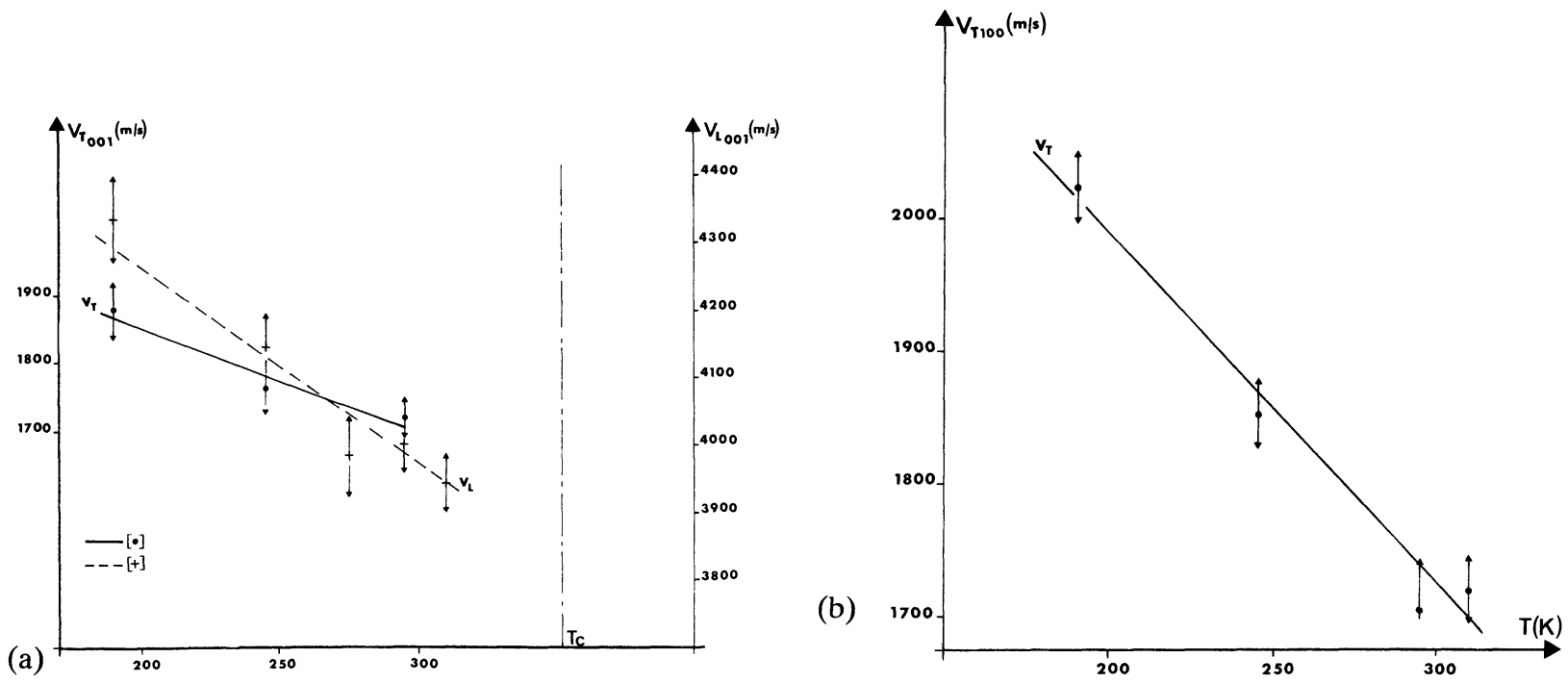

Fig. 8. - Variations avec la température des vitesses acoustiques. a) Suivant la direction $\mathbf{c}^{*}$. b) Suivant la direction $\mathbf{a}^{*}$. [Acoustic velocities versus temperature. a) Along $\mathbf{c}^{*}$. b) Along $\mathbf{a}^{*}$.] 
Tableau IV. - Symétries et mouvements associés en centre de zone.

[Symmetries and associated motions in the centre of Brillouin zone.]

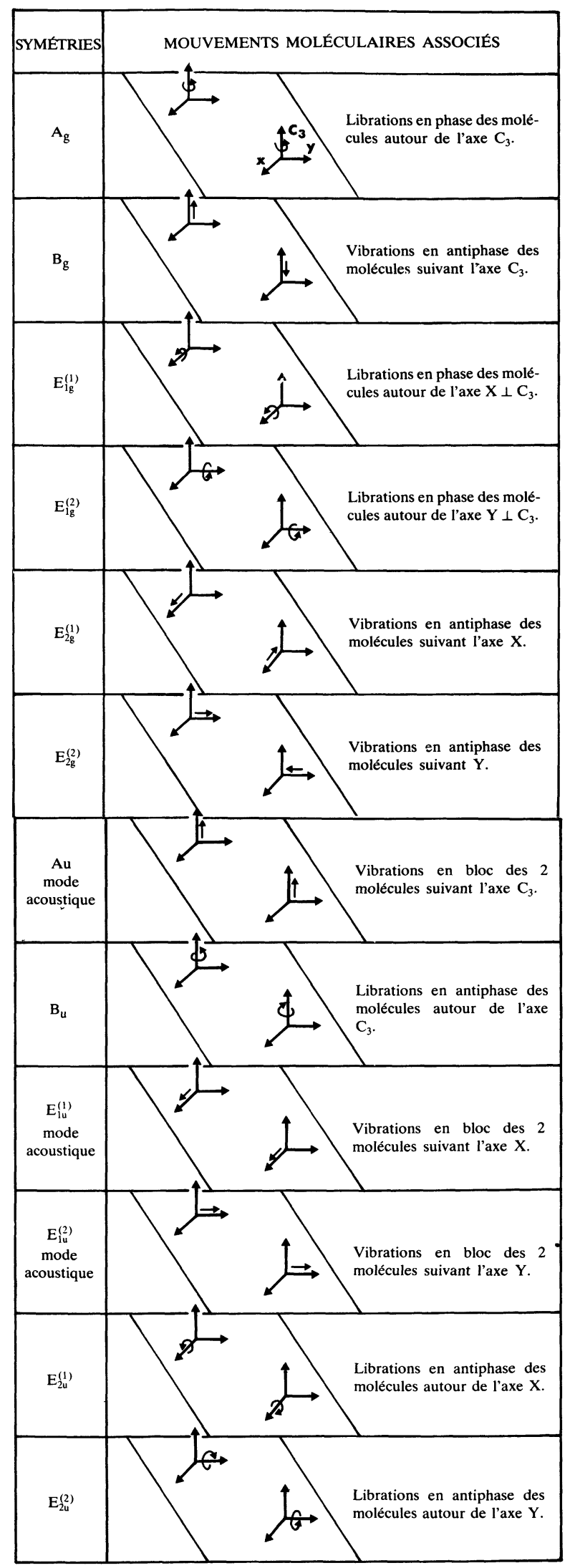

sont classiques pour un cristal moléculaire dans la mesure où elles traduisent un phénomène de dilatation thermique du composé. Cette hypothèse semble en accord avec la variation des paramètres sur le même intervalle de température (Fig. 9).

4.2 VARIATIONS AVEC LA TEMPÉRATURE DES FRÉQUENCES ET DES LARGEURS DES MODES OPTIQUES. L'autre objectif de cette étude était de compléter les résultats de diffusion Raman [3, 4], le problème posé étant le suivant :

la dépendance en température des largeurs des modes de libration actifs $\left(\mathrm{A}_{\mathrm{g}}, \mathrm{E}_{1 \mathrm{~g}}\right)$ montre un comportement semblant s'écarter à haute température de celui prévu par les théories anharmoniques $[9,10]$ contrairement au mode de translation $\mathrm{E}_{2 \mathrm{~g}}$ (annexe). Cette anomalie a été reliée à l'activation du désordre uniaxial à l'approche de $T_{\mathrm{c}}$ en accord avec le modèle de Smith [1] basé sur des résultats de R.M.N. et des calculs de potentiel de Reynolds [5].

Les mesures de diffusion neutronique ont eu pour but de vérifier cette hypothèse en apportant des renseignements à deux niveaux :

a) l'étude d'autres modes que ceux actifs en diffusion Raman. Une comparaison plus systématique peut être faite entre les modes de libration (autour de l'axe $\mathrm{C}_{3}$ et perpendiculaire à l'axe $\mathrm{C}_{3}$ ) et les modes de translation. (Pour mémoire nous donnons dans le tableau IV les types de mouvement associés à chaque mode de symétrie pour $\mathbf{q}=0$ ).

b) le comportement des largeurs en fonction de $\mathbf{Q}$ (vecteur de diffusion) et $\mathbf{q}$ (vecteur d'onde) afin d'évaluer éventuellement les parts respectives des effets liés aux corrélations réorientationnelles intermoléculaires de ceux liés au désordre.

4.2.1 Etude au centre de zone $(3 ; 0 ;-3)$. - Cette étude effectuée dans l'intervalle $190-310 \mathrm{~K}$ a permis une comparaison directe avec les mesures Raman correspondantes (modes $\mathrm{E}_{1 \mathrm{~g}}, \mathrm{E}_{2 \mathrm{~g}}, \mathrm{~A}_{\mathrm{g}}$; voir Tab. $\mathrm{V}$ et annexe). On note un accord satisfaisant entre les résultats issus des deux types d'expérience. De plus, conformément à la théorie, on observe que seul le mode $B_{u}$ s'élargit nettement dans cet intervalle de température (Fig. 9).

Tableau V. - Comparaison de l'évolution en température des fréquences obtenues par diffusion Raman et par diffusion neutronique.

[Comparison between Raman and neutron frequencies at different temperatures.]

\begin{tabular}{|c|c|}
\hline $\begin{array}{c}(\partial \nu / \partial T) \\
\text { par diffusion Raman }\end{array}$ & $\begin{array}{c}(\partial \nu / \partial T) \\
\text { par diffusion neutronique }\end{array}$ \\
\hline $\mathrm{E}_{1 \mathrm{~g}} \rightarrow-0,04 \mathrm{~cm}^{-1} \mathrm{~K}^{-1}$ & $\mathrm{E}_{2 \mathrm{u}} \rightarrow-0,038 \mathrm{~cm}^{-1} \mathrm{~K}^{-1}$ \\
$\mathrm{E}_{2 \mathrm{~g}} \rightarrow-0,06 \mathrm{~cm}^{-1} \mathrm{~K}^{-1}$ & $\mathrm{E}_{2 \mathrm{~g} \rightarrow-0,064 \mathrm{~cm}^{-1} \mathrm{~K}^{-1}}$ \\
$\mathrm{~A}_{\mathrm{g} \rightarrow-0,08 \mathrm{~cm}^{-1} \mathrm{~K}^{-1}}$ & $\mathrm{~B}_{\mathrm{u}} \rightarrow-0,082 \mathrm{~cm}^{-1} \mathrm{~K}^{-1}$ \\
\hline
\end{tabular}




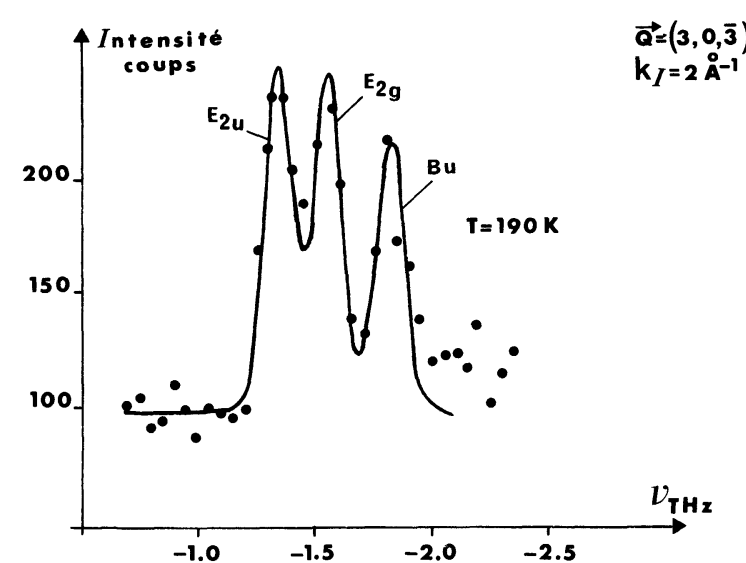

$\vec{Q}=(3,0, \overline{3})$
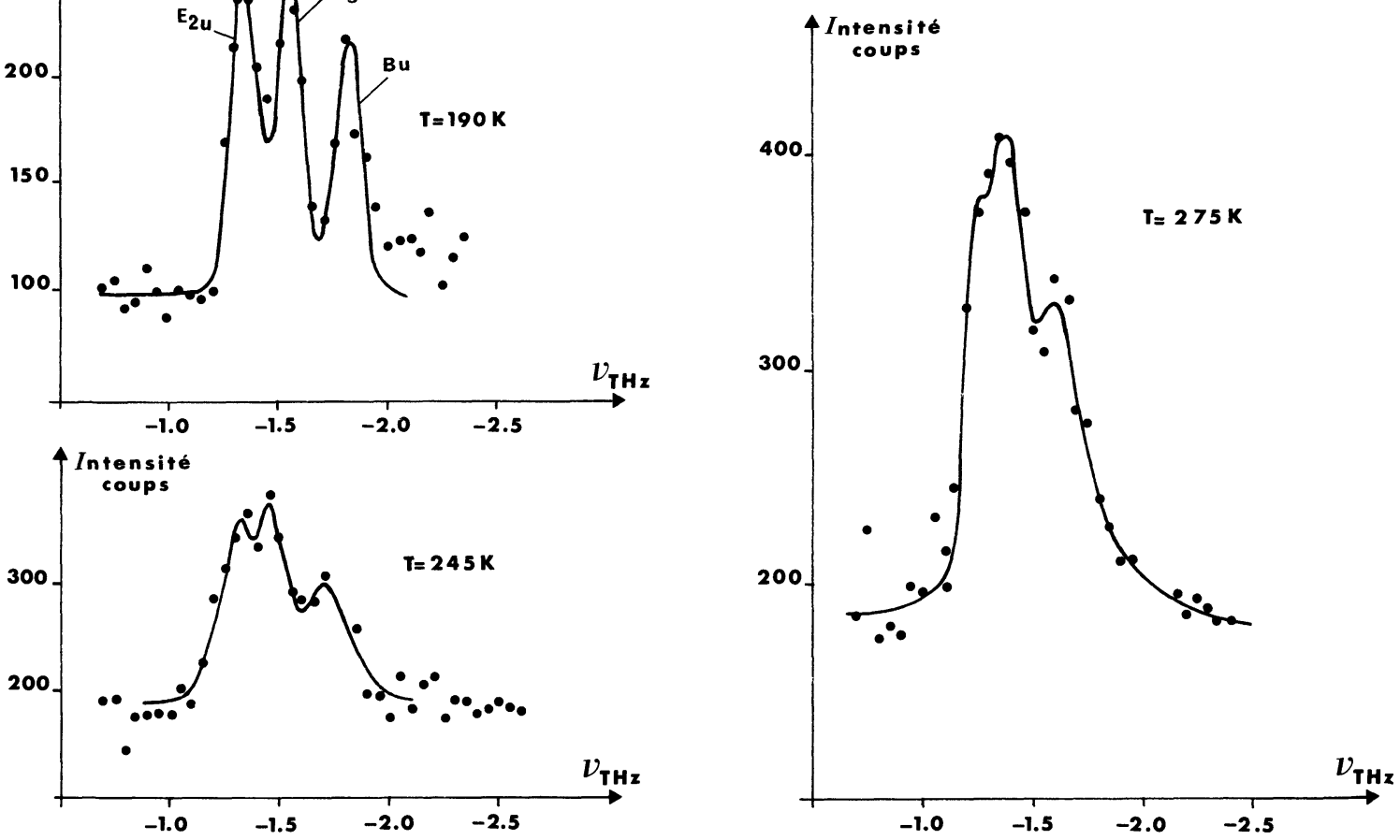

Fig. 9. - Evolution du groupe de phonons de centre de zone $(3 ; 0 ;-3)$.

[Evolution of the zone centre phonon group.]

4.2.2 Etude en des points intérieurs à la zone de Brillouin. - L'étude précédente a été poursuivie dans l'intervalle $190 \mathrm{~K} \rightarrow T_{\mathrm{c}}$ aux points $\mathbf{Q} 1=(1 ; 0$; $-4,2)$ et $\mathbf{Q} 2=(3 ; 0 ;-3,2)$ (Fig. 10). Concernant les modes de translation et les deux types de libration, elle a permis :

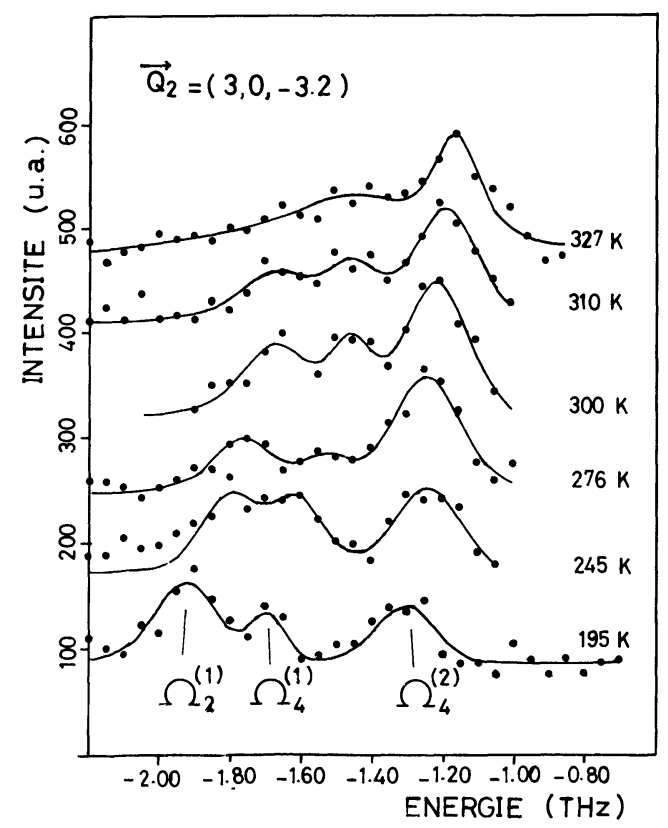

Fig. 10. - Phonons en $\mathbf{Q} 2$ en fonction de la température.

[Phonons groups at $\mathbf{Q} 2=(3 ; 0 ;-3.2)$.]
- d'une part, de confirmer le caractère quasi harmonique des premiers. Il s'agit aux points $\mathbf{Q} 1$ et Q2 du mode de symétrie $\Omega_{4}^{(2)}$. Ce mode (correspondant à $\mathrm{E}_{2 \mathrm{~g}}$ en centre de zone) est un exemple d'un mode à caractère translationnel quasi harmonique. On observe un comportement classique des variations des fréquences (Fig. 11) qu'on peut attribuer au seul effet de la dilatation thermique et des largeurs (Fig. 12a) en rapport avec une faible anharmonicité des mouvements ;

- d'autre part de mettre en évidence aux points Q1 et Q2 la forte dépendance avec la température des largeurs de certains modes de libration correspondant à des mouvements autour de l'axe $\mathrm{C}_{3}$ $\left(\Omega_{2}^{(1)}\right.$ par exemple) ainsi que celles d'autres modes correspondant à des librations autour d'axes perpendiculaires à l'axe $C_{3}\left(\Omega_{4}^{(1)}\right.$ par exemple). On notera toutefois que pour l'ensemble des modes étudiés la variation des fréquences avec la température est linéaire et cela jusqu'à la température de transition (Fig. 11). La figure 12a illustre la différence de comportement des largeurs avec la température entre un mode à caractère translationnel $\Omega_{4}^{(2)}$ et un mode à caractère librationnel $\Omega_{4}^{(1)}$; ce résultat est en accord avec celui obtenu à partir d'expériences de diffusion Raman concernant les modes $E_{1 \mathrm{~g}}$ et $\mathrm{E}_{2 \mathrm{~g}}$. On note une plus forte dépendance avec la température de la largeur du mode $\Omega_{4}^{(1)}$ par rapport à celle du mode $E_{1 \mathrm{~g}}$. Il semble que cet effet pourrait être lié à la fois à une variation avec $\mathbf{q}$ des largeurs et 


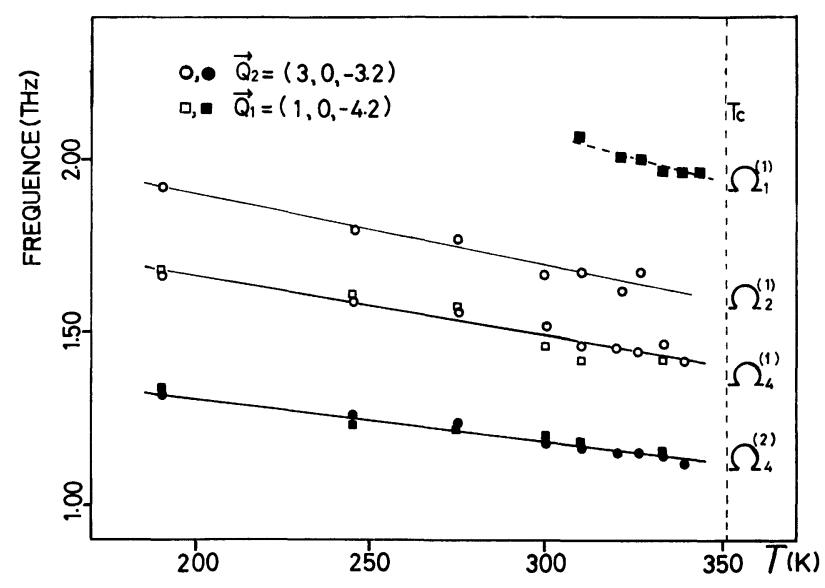

Fig. 11. - Variations avec la température des fréquences de phonons aux points $\mathbf{Q} 1=(1 ; 0 ;-4,2)$ et $\mathbf{Q} 2=$ $(3 ; 0 ;-3,2)$.

[Temperature dependence of phonon frequencies for $\mathbf{Q} 1$ and $\mathbf{Q} 2$ points.]
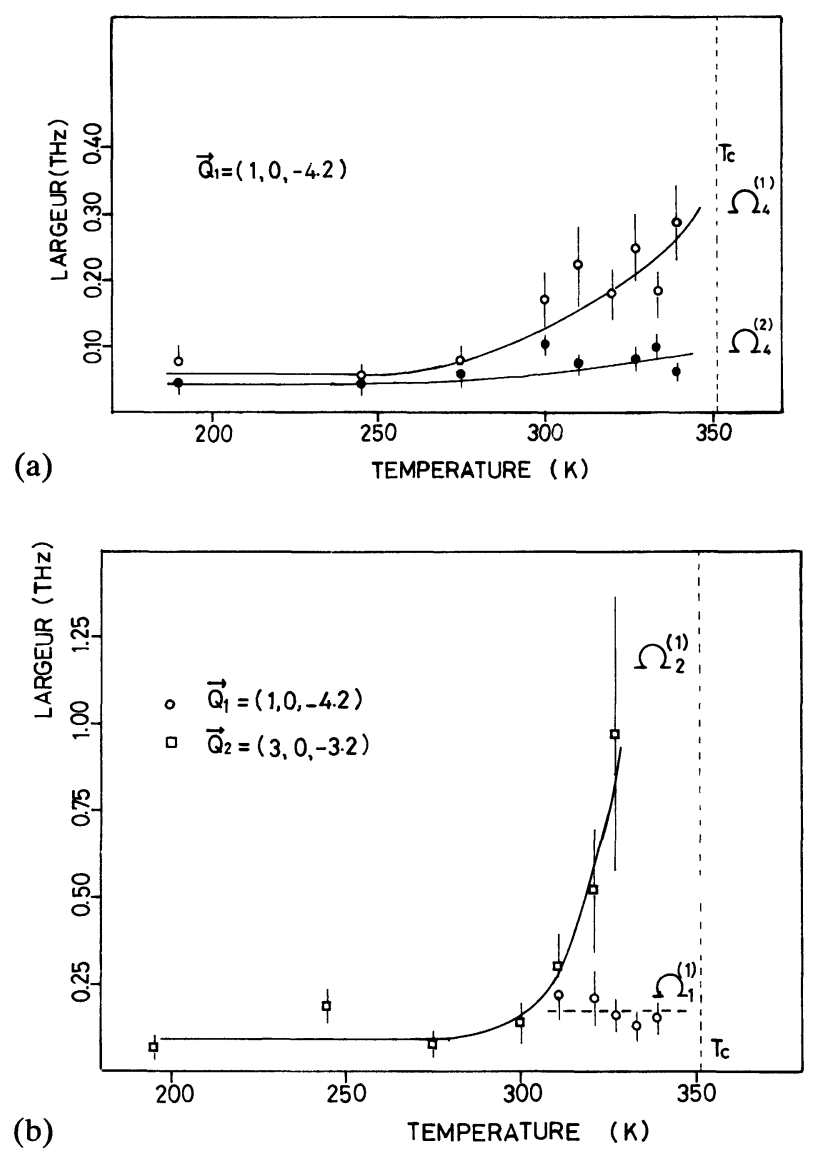

Fig. 12. - Evolution avec la température des largeurs de phonons en a) Q1, b) $\mathbf{Q} 2$ et $\mathbf{Q} 1$.

[Temperature evolution of phonon linewidths for the points : a) Q1, b) Q2 and Q1.]

aussi, pour des modes qui font intervenir des librations autour d'axes perpendiculaires à $C_{3}$, à une anharmonicité plus forte des mouvements en anti- phase que celle des mouvements en phase (Tab. IV).

La figure $12 \mathrm{~b}$ illustre d'une part la forte variation avec la température du mode $\Omega_{2}^{(1)}$ qui ne peut être décrite par des lois anharmoniques usuelles et d'autre part la différence de comportement entre les variations pour un même vecteur d'onde des modes $\Omega_{1}^{(1)}$ et $\Omega_{2}^{(1)}$ qui font tous deux intervenir des librations autour de l'axe $\mathrm{C}_{3}$ moléculaire, en phase dans le cas de $\Omega_{1}^{(1)}$ et en anti-phase pour $\Omega_{2}^{(1)}$ (Tab. IV). On remarquera la faible intensité de ce dernier mode (Fig. 10) et l'imprécision qui en résulte sur l'évaluation de sa largeur (Fig. 12b). La variation de la largeur de ce mode $\left(\Omega_{2}^{(1)}\right)$ est essentiellement due à haute température au couplage avec le désordre uniaxial qui, en accord avec les données de R.M.N. paraît s'établir en dessous de $T_{\mathrm{c}}$; en effet, comme il a déjà été signalé, les lois anharmoniques ne peuvent expliquer une aussi forte dépendance en température sur un domaine aussi réduit $(300 \mathrm{~K}$, $350 \mathrm{~K})$. De plus, dans le cas de processus simplement anharmoniques, il devrait en résulter une anomalie dans le comportement des fréquences, anomalie qui n'a pas été mise en évidence. Par contre, un effet de « temps de vie » de libration liée à la localisation des molécules du système dans des orientations données entre deux réorientations n'a d'influence essentielle que sur la largeur des modes et pas sur leur fréquence, les molécules librant alors dans un potentiel moyen.

Quant à la différence de comportement entre les modes $\Omega_{1}^{(1)}$ et $\Omega_{2}^{(1)}$ correspondant au même vecteur d'onde $\mathbf{q}(0,2$ u.r.) mais à des vecteurs de diffusion $\mathbf{Q}$ différents $\left(|\mathbf{Q} 2|=4,17 \AA^{-1}\right.$ pour $\Omega_{2}^{(1)},|\mathbf{Q} 1|=$ $3,06 \AA^{-1}$ pour $\Omega_{1}^{(1)}$ ) on peut en donner deux explications possibles. La première consiste à dire qu'à cause du désordre les règles habituelles de sélection sur les vecteurs d'onde disparaissent donnant lieu à un élargissement d'autant plus grand que $|\mathbf{Q}|$ est grand. Une deuxième explication est cependant possible. Au centre de zone le mode $\mathrm{A}_{\mathrm{g}}$ correspond à une rotation en phase des molécules autour de $C_{3}$ alors que $B_{u}$ est une rotation en anti-phase et on a déjà mentionné que $B_{u}$ était élargi. Il est possible de voir à partir de la figure 2 que le mouvement en antiphase est le moins gêné stériquement. Il peut en résulter une plus forte anharmonicité de ce type de mouvement.

La même remarque peut être faite à propos des mouvements de libration en phase et en anti-phase autour d'axes perpendiculaires à $\mathrm{C}_{3}$. Ceci pourrait expliquer la plus forte anharmonicité de ceux-ci (mode $\Omega_{4}^{(1)}$ par exemple) par rapport à ceux-là (mode $\mathrm{E}_{1 \mathrm{~g}}$ ) que nous avons signalé précédemment.

4.2.3 Etude au bord de zone $\mathbf{Q 3}=(0 ; 0 ;-4,5)$ (Fig. 13). - L'étude au bord de zone (point Q3) présente plusieurs avantages. La dégénérescence de 


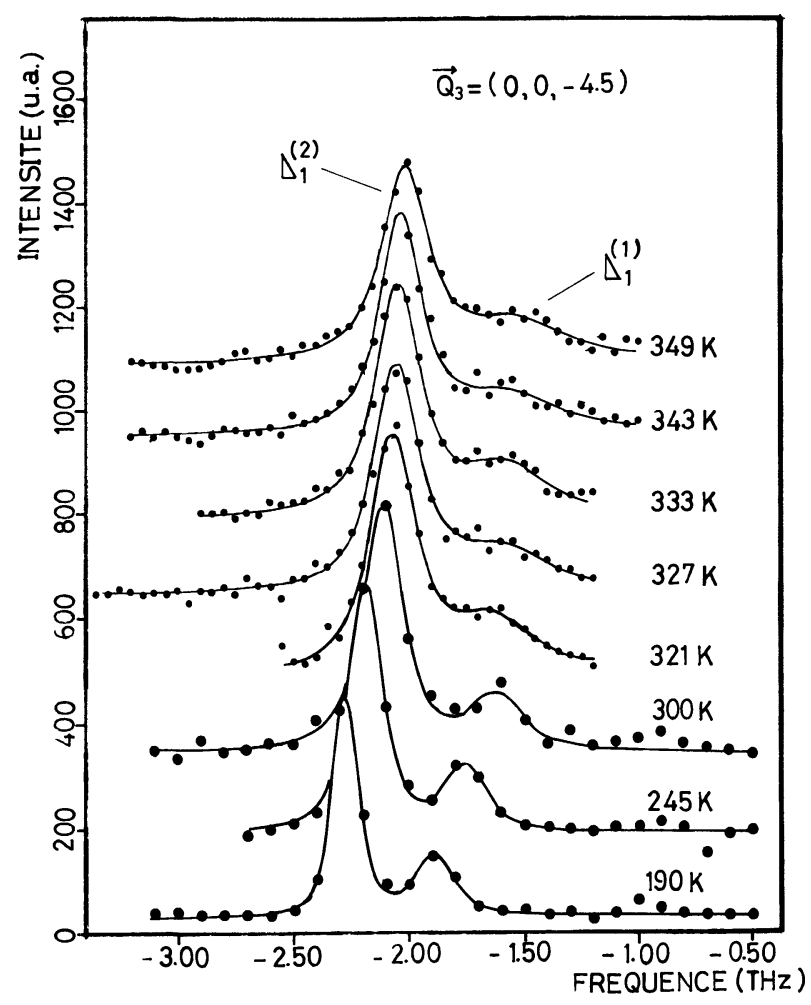

Fig. 13. - Phonons en $\mathbf{Q} 3$ en fonction de la température.

[Phonons groups at Q3 $=(0 ; 0 ;-4.5)$.]

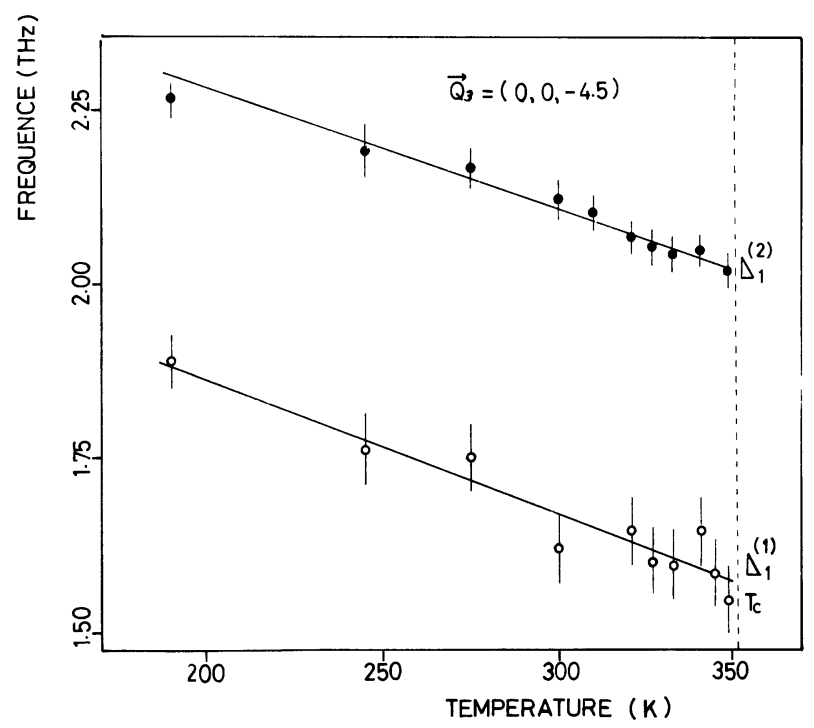

Fig. 14. - Variations avec la température des fréquences de phonons au point $\mathbf{Q} 3=(0 ; 0 ;-4,5)$.

[Temperature dependence of phonon frequencies for Q3.]

certains modes qui s'y produit rend moins complexe le traitement des spectres par le simple fait que moins de modes se chevauchent dans un même intervalle de fréquence et aussi parce que l'intensité des modes est sensiblement plus forte (Fig. 13). Les variations des fréquences et des largeurs du mode $\Delta_{1}^{(1)}$ qui a un caractère librationnel (libration autour de $\mathrm{C}_{3}$ ) et du mode $\Delta_{1}^{(2)}$ qui a un caractère translationnel (le long de $\mathrm{C}_{3}$ ) sont représentés sur les figures 14 et 15. On observe, en accord avec la description précédente, l'effet de l'apparition du désordre uniaxial par l'évolution avec la température des largeurs des modes relatifs aux librations autour de l'axe $\mathrm{C}_{3}$ moléculaire.

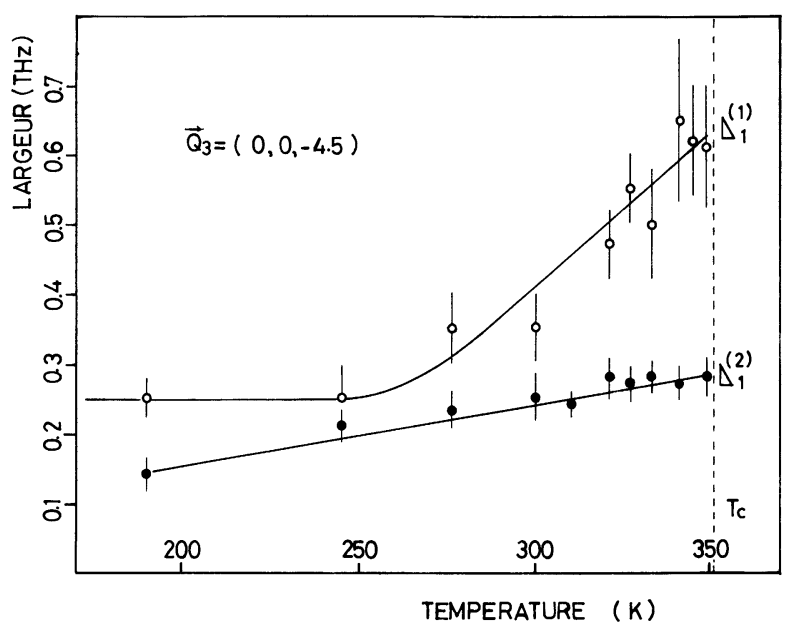

Fig. 15. - Evolution avec la température des largeurs de phonons au point $\mathbf{Q 3}$.

[Temperature evolution of phonon linewidths for Q3.]

\section{Conclusion.}

Les expériences de diffusion neutronique présentées dans cet article ont permis la mesure de la presque totalité des courbes de dispersion suivant c* à $^{*}$ 3 températures. Les données collectées à la température ambiante ont été utilisées dans l'ajustement d'un modèle quasi harmonique qui restitue avec un accord satisfaisant les courbes expérimentales.

De plus l'étude des groupes de phonons aux points $\mathbf{Q} 1, \mathbf{Q} 2$ et $\mathbf{Q} 3$ ont permis de compléter les résultats de diffusion Raman à plusieurs niveaux :

- les largeurs des phonons pour des vecteurs $\mathbf{q}$ différents de zéro appartenant à des branches de symétrie associée à des mouvements de nature similaire, révèlent des comportements dynamiques différents. Aux points $\mathbf{Q} 1$ et $\mathbf{Q} 2\left(\mathbf{q}=-0,2 \mathbf{c}^{*} / 2\right)$ les fréquences des modes $\Omega_{1}^{(1)}\left(\mathrm{A}_{\mathrm{g}}\right.$ en centre de zone) et $\Omega_{2}^{(1)}\left(\mathrm{B}_{\mathrm{u}}\right.$ en centre de zone) ont des variations semblables alors que la dépendance en température de la largeur du mode $\Omega_{2}^{(1)}$ est beaucoup plus forte que celle du mode $\Omega_{1}^{(1)}$. Cet effet est à mettre en parallèle avec la moins grande gêne stérique pour les mouvements en anti-phase autour de l'axe $\mathrm{C}_{3}$ (mode $\left.\Omega_{2}^{(1)}\right)$ que pour les mouvements en phase (mode $\Omega_{1}^{(1)}$ ) avec deux conséquences: une plus grande anharmonicité des premiers mouvements cités et un couplage direct avec le désordre uniaxial qui 
commence à s'établir via la grande amplitude des mouvements de libration en anti-phase.

Un modèle de dynamique anharmonique classique pourrait fournir davantage de renseignements. Cependant sa mise en œuvre n'est possible qu'à partir de données cristallographiques précises qu'il paraît difficile d'obtenir au voisinage de $T_{\mathrm{c}}$. Une autre approche théorique, basée sur un développement du potentiel en fonctions adaptées à la symétrie est actuellement envisagée. Elle permettrait une description plus judicieuse des mouvements moléculaires à l'approche de $T_{\mathrm{c}}$.

\section{Remerciements.}

Les auteurs remercient Madame Belloc du L.A.S.I.R. pour sa participation prépondérante à la réalisation de la deutériation de la triéthylènediamine.

\section{Annexe.}

EVOLUTION EN TEMPÉRATURE DES MODES RAMAN EN CENTRE DE ZONE. - Les figures Aa, b, c représentent la variation des largeurs des modes Raman en fonction de la température. Pour chacun des trois modes, la courbe en trait plein est tracée à partir des points expérimentaux alors que celle en trait discontinu correspond à l'ajustement des points expérimentaux par les lois théoriques définies cidessous :

a) La largeur est ajustée à la loi :

$$
\Gamma_{\mathrm{A}_{\mathrm{g}}}(T)=a_{\mathrm{Ag}_{\mathrm{g}}} T+b_{\mathrm{A}_{\mathrm{g}}} T^{2}+c_{\mathrm{A}_{\mathrm{g}}} T^{3}
$$

avec

$$
\begin{aligned}
& a_{\mathrm{A}_{\mathrm{g}}}=10^{-3} \mathrm{~cm}^{-1} \mathrm{~K}^{-1} \\
& b_{\mathrm{A}_{\mathrm{g}}}=1,5 \times 10^{-5} \mathrm{~cm}^{-1} \mathrm{~K}^{-2} \\
& c_{\mathrm{A}_{\mathrm{g}}}=5 \times 10^{-8} \mathrm{~cm}^{-1} \mathrm{~K}^{-3} .
\end{aligned}
$$

b) La même loi que précédemment est employée avec :

$$
\begin{aligned}
& a_{\mathrm{E}_{1 \mathrm{~g}}}=0,011 \mathrm{~cm}^{-1} \mathrm{~K}^{-1} \\
& b_{\mathrm{E}_{1 \mathrm{~g}}}=1,6 \times 10^{-5} \mathrm{~cm}^{-1} \mathrm{~K}^{-2} \\
& c_{\mathrm{E}_{1 \mathrm{~g}}}=7,5 \times 10^{-9} \mathrm{~cm}^{-1} \mathrm{~K}^{-3} .
\end{aligned}
$$

c) La loi est maintenant :

$$
\Gamma_{\mathrm{E}_{2 \mathrm{~g}}}=B_{\mathrm{E} 2 \mathrm{~g}}\left[2 n\left(\nu_{\mathrm{E} 2 \mathrm{~g}} / 2, T\right)+1\right]
$$

Ou

$$
\nu_{\mathrm{E}_{2 \mathrm{~g}}}(T)=\nu_{\mathrm{E}_{2 \mathrm{~g}}}(0)-A_{\mathrm{E}_{2 \mathrm{~g}}}\left[n\left(\nu_{\mathrm{E}_{2 \mathrm{~g}}}, T\right)+1 / 2\right]
$$
avec

$$
\begin{aligned}
& B_{\mathrm{E}_{2 \mathrm{~g}}}=0,14 \mathrm{~cm}^{-1} \\
& A_{\mathrm{E}_{2 \mathrm{~g}}}=3,80 \mathrm{~cm}^{-1} \\
& \nu_{\mathrm{E}_{2 \mathrm{~g}}}=62,00 \mathrm{~cm}^{-1} .
\end{aligned}
$$

Cette loi traduit le fait que l'annihilation d'un phonon optique correspond à la création de deux phonons acoustiques de fréquence moitié.

$n(\nu, T)$ est le facteur de population.

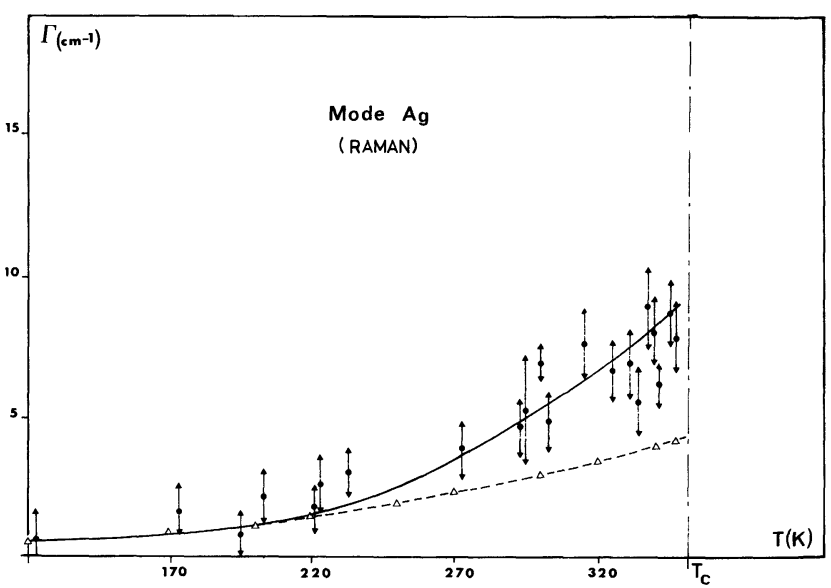

Fig. Aa.

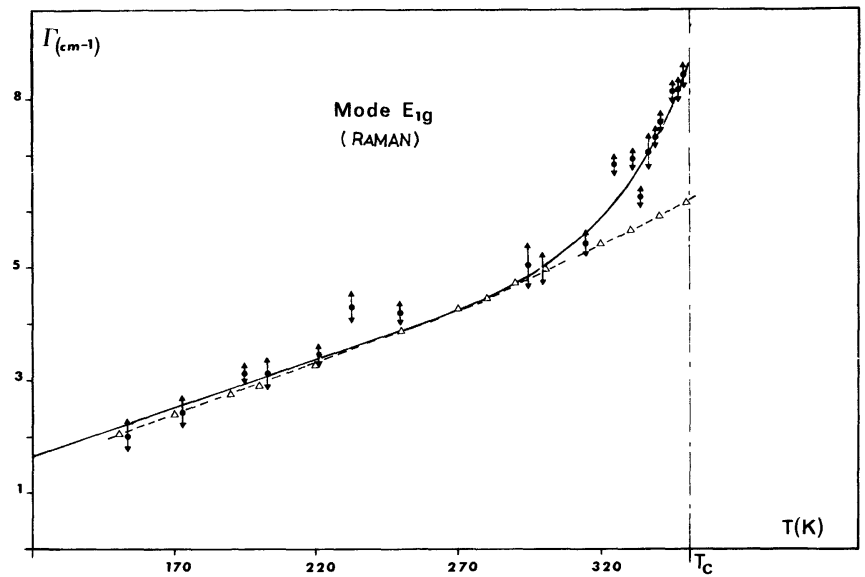

Fig. Ab.

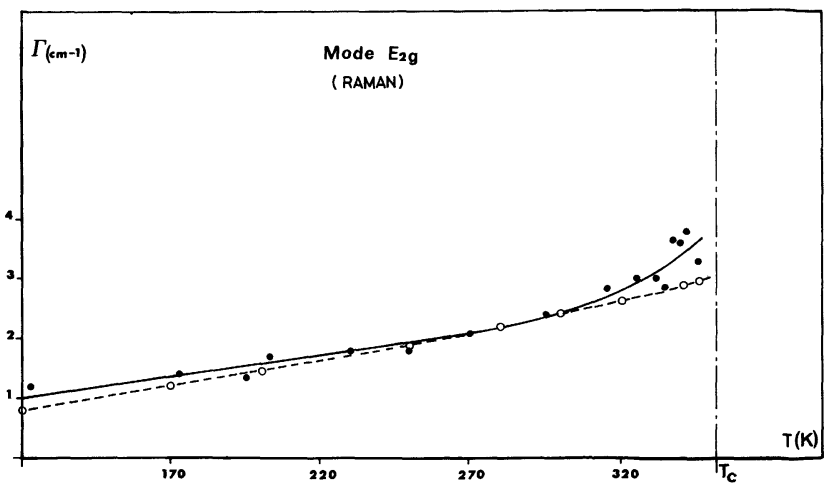

Fig. Ac. 


\section{Bibliographie}

[1] Smith, G. W., J. Chem. Phys. 43 (1965) 4325-4336.

[2] Sauvajol, J. L., J. Phys. C 13 (1980) L927.

[3] Sauvajol, J. L., J. Raman Spectrosc. 13 (1982) 270.

[4] Hedoux, A., Thèse Université de Lille I (1986).

[5] Reynolds, P. A., Mol. Phys. 28 (1974) 633-652.

[6] Hennion, B., Hennion, M., Hippert, F. et Murani, A. P., J. Phys. F 14 (1984) 489-504.
[7] Reynolds, P. A., J. Chem. Phys. 59 (1973) 27772786.

[8] Venkataraman, G. et Sahni, V. C., Rev. Mod. Phys. 42 (1970) 409.

[9] Kalus, J., J. Chim. Phys. 82 (1985) 137.

[10] Lefebvre, J., Thèse Université de Lille I (1976). 